Cahiers
de a Recherche
sur les Droits

Cahiers de la recherche sur les droits fondamentaux

15 | 2017

Le corps humain saisi par le droit : entre liberté et propriété

\title{
Le statut du donneur
}

The Status of the Donor

Antoine Tadros

\section{(2) OpenEdition}

Journals

Édition électronique

URL : https://journals.openedition.org/crdf/546

DOI : $10.4000 /$ crdf.546

ISSN : 2264-1246

Éditeur

Presses universitaires de Caen

Édition imprimée

Date de publication : 1 novembre 2017

Pagination : 45-53

ISBN : 978-2-84133-858-0

ISSN : $1634-8842$

Référence électronique

Antoine Tadros, "Le statut du donneur », Cahiers de la recherche sur les droits fondamentaux [En ligne],

15 | 2017, mis en ligne le 01 octobre 2019, consulté le 14 novembre 2022. URL : http://

journals.openedition.org/crdf/546 ; DOI : https://doi.org/10.4000/crdf.546 


\title{
Le statut du donneur
}

\author{
Antoine TADROS \\ Professeur de droit privé à l'université de Picardie-Jules-Verne
}

I. La tentative infructueuse de rattachement du statut du donneur aux prérogatives individuelles

A. Les opérations autorisées au donneur

B. Les modalités des opérations autorisées au donneur

II. La mise à l'épreuve du paradigme reposant sur l'intérêt général

A. Un compromis hasardeux

B. La liberté et la propriété: des instruments de correction aujourd'hui, des matrices du statut du donneur demain?

Le statut du donneur relève-t-il davantage de la liberté ou de la propriété? La question est à la fois plus simple et plus complexe que lorsqu'on la pose à propos du corps humain en son entier ${ }^{1}$.

Elle est d'abord plus simple. Elle est plus simple parce que le champ d'investigation est plus restreint que le corps humain. Lorsqu'on aborde le corps humain en son entier, la difficulté pour trancher entre la liberté et la propriété tient essentiellement au fait que le corps humain et la personnalité juridique sont $a$ priori consubstantiels ${ }^{2}$; il faut faire un véritable effort d'abstraction pour parvenir à distinguer la personne juridique de la personne physique ${ }^{3}$. Mais cet effort n'est plus nécessaire lorsqu'on aborde le statut du donneur parce que le statut du donneur renvoie à l'hypothèse dans laquelle une personne détache un élément de son corps pour le faire circuler: on donne son rein, son poumon, son sang, ses gamètes, ses cheveux, ses dents, etc. En d'autres termes, on donne une pièce détachée.

Ce détachement permet de créer une distanciation entre le corps humain, socle de la personnalité juridique, et l'élément qui va circuler. Dit autrement, le rein, le poumon, le sang de la personne ne sont pas la personne ${ }^{4}$. Si ces éléments ne peuvent être confondus avec la personne, que sont-ils? Conformément au découpage du Code civil, tout ce qui n'est pas personne est chose, et le fondement de la circulation des choses entre les personnes est la propriétés. Ainsi, à propos du statut du donneur, l'altérité, la faculté de distinguer entre la personne et l'élément qu'elle va faire circuler fait inéluctablement pencher la balance en faveur de la propriété.

1. Eu égard au thème général du colloque dans le cadre duquel cette contribution a été rédigée.

2. Voir en ce sens S.-M. Ferrié, Le droit à l'autodétermination de la personne humaine. Essai en faveur du renouvellement des pouvoirs de la personne sur son corps, thèse de doctorat, université Paris 1 - Panthéon-Sorbonne, 2015, $\mathrm{n}^{\circ} 112$.

3. Voir T. Revet, «Le corps humain est-il une chose appropriée?», Revue trimestrielle de droit civil, 2017, p. 587 sq. Voir aussi S.-M. Ferrié, Le

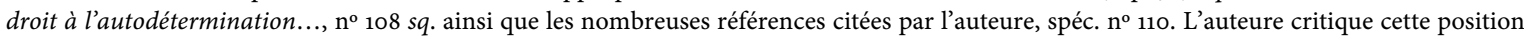
et considère qu'il n'est pas possible de distinguer entre la personnalité juridique et le corps humain qui représente son enveloppe charnelle.

4. Voir A.-B. Caire, "Le corps gratuit: réflexions sur le principe de gratuité en matière d'utilisation de produits et d'éléments du corps humain", Revue de droit sanitaire et social, 2015, p. 865 sq.

5. Voir notamment G. Cornu, Droit civil. Les biens, $13^{\mathrm{e}}$ éd., Paris, Montchrestien (Domat droit privé), 2007, $\mathrm{n}^{\circ} 1$ : «S’il est vrai qu'en ce monde rivalisent deux ordres de valeurs irréductibles - l'argent et l'amour - l'ordre juridique en porte un peu le reflet lorsqu'il présente le droit patrimonial et le droit extrapatrimonial»; voir aussi W. Dross, Droit civil. Les choses, Paris, LGDJ, 2012, $\mathrm{n}^{\circ}$ 1: «La distinction des personnes et des choses est totalisante: une chose est tout ce qui n'est pas une personne». 
Voilà pourquoi la question de savoir si le statut du donneur relève davantage de la liberté ou de la propriété semble plus simple que celle de savoir si le corps humain en son entier relève de la liberté ou de la propriété. La réduction du champ d'analyse paraît ainsi faciliter la tâche. Toutefois, c'était sans compter sur la réglementation du statut du donneur. La confrontation entre la liberté individuelle ou la propriété des éléments du corps humain à la réglementation applicable au don de substance humaine plonge l'analyste dans un abîme de perplexité. La question devient alors extrêmement complexe.

Pourquoi une telle complexité ? Le législateur a fortement encadré le statut du donneur. Pour avoir une idée de ce qu'un individu peut faire avec un élément de son corps, il faut consulter le Code civil bien sûr, les articles 16-1 et suivants; il faut également consulter le Code de la santé publique, les articles L. 1211-1 et suivants pour les principes généraux applicables au don de matière humaine, puis ceux qui viennent à la suite, lesquels, en fonction de l'élément donné - organe, sang, tissus, cellules, gamète -, prévoient un régime particulier. En somme tout est réglementé: la finalité du prélèvement de la matière humaine, le consentement du donneur, l'anonymat et la gratuité du don, les établissements autorisés à réaliser le prélèvement, la mise en place de comités médicaux dédiés à la question, etc.

Cet encadrement strict et précis du statut du donneur n'est pas neutre lorsqu'on s'interroge sur le point de savoir si le statut du donneur relève de la propriété ou de la liberté. La liberté et la propriété partagent un élément fondamental en commun. Ces deux concepts ont été élaborés en 1789 comme des prérogatives individuelles absolues ${ }^{6}$. La liberté comme la propriété ne sont pas des autorisations ponctuelles, mais des autorisations de principe. Si l'on doit revenir à des définitions simples et consensuelles: la liberté est la faculté de faire tout ce qui ne nuit pas à autrui ${ }^{7}$; la propriété n'est autre que le concept de liberté appliqué aux choses, elle est le droit de faire d'un bien tout ce qui n'est pas interdit par la loi ou les règlements ${ }^{8}$.

Autrement dit, la liberté comme la propriété n'existent que si le législateur n'intervient que ponctuellement pour interdire tel ou tel comportement en ce qui concerne la liberté, tel ou tel usage d'un bien en ce qui concerne la propriété. Et ces interdictions ponctuelles sont toujours guidées par le même impératif: l'intérêt général.
Ainsi, en amont, avant de se demander si le statut du donneur relève davantage de la propriété ou de la liberté, il faut vérifier que la réglementation du donneur peut effectivement être regardée comme une série de restrictions ponctuelles à une prérogative individuelle sur les éléments du corps humain qui serait motivée par l'intérêt général.

C'est à ce propos que l'analyse devient extrêmement compliquée. L'intérêt général en matière de don de matière humaine est très présent. On peut identifier deux grandes déclinaisons de l'intérêt général. Il y a tout d'abord l'inviolabilité du corps humain, le respect qui lui est dû, la dignité humaine qui ont été proclamés dans le Code civil ${ }^{9}$. Il y a ensuite l'intérêt scientifique et thérapeutique mentionné tout au long des dispositions du Code de la santé publique parce que le don de substance humaine permet de sauver la vie ou de donner la vie ${ }^{10}$. Ces deux grands intérêts généraux identifiés, un constat s'impose: toute la réglementation du statut du donneur est tournée vers la recherche d'un compromis entre les deux.

Dès lors qu'il est dans l'intérêt scientifique et thérapeutique que les éléments du corps humain circulent, il faut permettre leur circulation, mais cette circulation doit être circonscrite au nom de l'inviolabilité du corps humain, du respect dû au corps, de la dignité humaine. Un organe doit pouvoir passer d'un corps humain à un autre pour sauver la vie du receveur. L'intérêt thérapeutique commande que l'organe puisse circuler. Néanmoins l'organe ne peut être vendu car cela occasionnerait une marchandisation des éléments du corps humain, ce que la dignité humaine ne saurait tolérer. De même, l'organe ne peut être prélevé contre le gré du donneur, le principe d'inviolabilité du corps humain s'y opposerait.

Dans un tel schéma organisé autour des restrictions, quelle place reste-t-il pour les prérogatives individuelles? La liberté individuelle et la propriété n'interviennent plus que de manière accidentelle. Elles sont évoquées bien plus que proclamées. Certes, intuitivement, le don fait penser à la donation qui suppose que le donneur soit propriétaire de la chose donnée; certes, intuitivement, on est tenté de voir dans le consentement du donneur l'expression de la liberté contractuelle. Toutefois, derrière les mots, on peine à retrouver les concepts.

Dans une société qui a placé la liberté individuelle au cœur de son système juridique, le statut du donneur tel

6. Il suffit ici de rappeler les dispositions de l'article 2 de la Déclaration des droits de l'homme et du citoyen de 1789 (DDHC): «Le but de toute association politique est la conservation des droits naturels et imprescriptibles de l'Homme. Ces droits sont la liberté, la propriété, la sûreté, et la résistance à l'oppression».

7. Art. 4 de la DDHC: «La liberté consiste à pouvoir faire tout ce qui ne nuit pas à autrui : ainsi, l'exercice des droits naturels de chaque homme n'a de bornes que celles qui assurent aux autres Membres de la Société la jouissance de ces mêmes droits. Ces bornes ne peuvent être déterminées que par la Loi».

8. Art. 544 du Code civil: «La propriété est le droit de jouir et disposer des choses de la manière la plus absolue, pourvu qu'on n'en fasse pas un usage prohibé par les lois ou par les règlements".

9. Le principe est aujourd'hui affirmé à l'article $16 \mathrm{du}$ Code civil: «La loi assure la primauté de la personne, interdit toute atteinte à la dignité de celle-ci et garantit le respect de l'être humain dès le commencement de sa vie».

10. Art. L. 1211-1 du Code de la santé publique: «La cession et l'utilisation des éléments et produits du corps humain sont régies par les dispositions du chapitre II du titre $\mathrm{I}^{\mathrm{er}} \mathrm{du}$ livre $\mathrm{I}^{\text {er }}$ du code civil et par les dispositions du présent livre./Les activités afférentes à ces éléments et produits, mentionnées au présent livre, y compris l'importation et l'exportation de ceux-ci, doivent poursuivre une fin médicale ou scientifique, ou être menées dans le cadre de procédures judiciaires conformément aux dispositions applicables à celles-ci». 
qu'il est prévu en droit positif s'acclimate mal aux grands principes parce qu'il renverse le paradigme établi en 1789 . Il n'est pas question en la matière de prérogatives individuelles - liberté ou propriété - qui seraient tempérées par des exceptions motivées par l'intérêt général.

L'intérêt général est la matrice du statut du donneur. Le statut du donneur met en scène deux intérêts collectifs contradictoires: le respect dû au corps humain d'un côté et l'intérêt scientifique ou thérapeutique de l'autre. La liberté de manière générale ne semble plus intervenir qu'à la marge.

Un tel changement de paradigme permet de faire un constat et de poser une question. En premier lieu, il permet de faire un constat: toute tentative de rattachement du statut du donneur à une prérogative individuelle - qu'il s'agisse de la propriété ou de la liberté - s'avère infructueuse (I). En second lieu, concevoir l'intérêt général comme la matrice de la réglementation du statut du donneur conduit-il a une législation efficiente? Si la réponse est positive, il convient de laisser de côté la liberté et la propriété; si la réponse est négative, alors on peut s'interroger sur l'éventualité de faire table rase de la réglementation existante et de reconstruire le régime du statut du donneur à partir de la liberté individuelle (II).

\section{La tentative infructueuse de rattachement du statut du donneur aux prérogatives individuelles}

L'engouement pour les prérogatives individuelles mérite d'être mis à l'épreuve. La question qu'il convient de poser est simple: sont-elles restreintes en matière de don de substance humaine, auquel cas elles sont de droit positif, ou alors le législateur dicte-t-il le comportement que l'individu peut adopter, auquel cas les prérogatives individuelles doivent être considérées hors du champ du statut du donneur? À l'analyse, ces prérogatives individuelles sont hors du champ du statut du donneur. La personne n'a pas d'emprise sur les opérations qu'elle peut effectivement accomplir à l'égard des éléments de son corps (A). Elle n'a pas davantage d'emprise sur les modalités de ces opérations (B).

\section{A. Les opérations autorisées au donneur}

La destination des éléments du corps humain. La liberté comme la propriété postulent une autorisation de principe accordée par le droit à l'individu dans la seule limite de restrictions fondées sur l'intérêt général ${ }^{11}$. Appliqué au donneur, cet axiome commande que ce dernier puisse choisir la destination des éléments de son corps. La destination d'une chose, en l'occurrence d'un élément du corps, correspond au but auquel la personne affecte l'élément et aux moyens permettant de réaliser ce but ${ }^{12}$. Or, le droit positif ne laisse aucune marge de manœuvre quant à l'emprise de la volonté de l'individu, que ce soit sur le but ou sur les moyens d'y parvenir.

L'absence de choix quant au but. Choisir le but auquel la personne peut affecter un élément de son corps, c'est pouvoir opter, parmi les nombreux services que l'élément du corps peut rendre, pour celui qu'elle souhaite. Or, tel n'est pas le cas en droit positif. Quelques exemples permettent de l'illustrer aisément. Peut-on imaginer l'ouverture d'un restaurant dans lequel une personne accepterait de cuisiner avec son sang? Est-il possible de donner sa peau à un ami qui a le même teint pour qu'il puisse dissimuler un tatouage qu'il n'apprécie plus? Sans dresser une liste à la Prévert, prenons un dernier exemple: peut-on imaginer qu'une personne décide de mettre son rein, une partie de son foie ou encore des gamètes au service d'une exposition d'art moderne après sa mort? La convention qui l'envisagerait est-elle valable? L'hypothèse évoque immédiatement l'affaire "Our body» dans laquelle la Cour de cassation a considéré que l'exposition de cadavres à des fins commerciales méconnaissait le respect dû au corps humain même après la mort. Cette solution est fondée sur l'article 16-1-1, alinéa 2 du Code civil ${ }^{13}$. Ce qui est valable pour le traitement du corps post mortem l'est a fortiori au cours de la vie. En effet, l'article 16-1-1 indique que "[1]e respect dû au corps humain ne cesse pas avec la mort»; il renvoie ainsi à l'article 16-1 qui le précède et indique que " $[\mathrm{c}]$ hacun a le droit au respect de son corps» de son vivant. Mutatis mutandis, et pour reprendre l'exemple évoqué, il faut en conclure qu'une personne ne peut pas de son vivant faire circuler un organe pour les besoins d'une exposition.

Est-ce suffisant pour écarter toute idée de liberté ou de propriété du donneur sur les éléments de son corps? Certainement non. La liberté comme la propriété sont affectées de limites. La loi peut ainsi ponctuellement - mais seulement ponctuellement - interdire tel ou tel but auquel le donneur souhaiterait employer un élément de son corps. Toutefois, en la matière, le législateur ne se contente pas de prévoir des interdictions qui viendraient confirmer le principe selon lequel un individu peut laisser libre cours à sa volonté lorsqu'il est question des éléments de son corps. Tout au contraire, la loi définit de manière limitative les buts pour lesquels elle autorise la circulation d'un organe, de tissus ou encore du sang humain. C'est dire que ces

11. Voir supra.

12. Sur la notion de destination, voir S. Guinchard, L'affectation des biens en droit privé français, préface de R. Nerson, Paris, LGDJ, 1976, $\mathrm{n}^{\circ}$ 423; S. Becquet, Le bien industriel, préface de T. Revet, Paris, LGDJ, 2005, $\mathrm{n}^{\circ} 19$; E. Dockès, "Essai sur la notion d'usufruit », Revue trimestrielle de droit civil, 1995, p. 479, spéc. n 19: l'auteur illustre son propos à l'aide d'un morceau de bois et indique que «[l]e propriétaire d'un morceau de bois pourra décider notamment, de le brûler, de le sculpter ou d'en faire un tuteur. Ce choix est aussi celui d'un but assigné au bien. Il s'agit, à l'aide du morceau de bois en question soit de se chauffer, soit de satisfaire un besoin esthétique, soit encore d'aider une plante à pousser verticalement ".

13. Cass., $1^{\text {re }}$ civ., 16 septembre 2010, $\mathrm{n}^{\circ}$ 09-67.456: La semaine juridique, édition générale, $\mathrm{n}^{\circ} 50,13$ décembre 2010, 1239, note $\mathrm{B}$. Marrion. 
finalités sont restrictives. En effet, qu'il s'agisse du Code civil ou du Code de la santé publique, la circulation de ces éléments n'y est envisagée que dans deux hypothèses spécifiques: le but doit être thérapeutique ou scientifique ${ }^{14}$. En dehors de ces hypothèses, l'opération par laquelle une personne met en circulation un des éléments de son corps doit être considérée comme illicite.

Si l'individu n'a pas la possibilité de choisir le service qu'il entend tirer d'un élément de son corps, peut-on encore tenter de découvrir un îlot de liberté ou de propriété quant aux moyens qu'il peut mettre en œuvre pour parvenir aux buts définis par la loi?

L'absence de choix quant aux moyens. Là encore un constat identique s'impose: l'individu ne peut choisir le moyen par lequel son organe, son sang ou ses tissus vont circuler, et ce même s'il se conforme aux objectifs assignés par la loi à la circulation de ces éléments. Les termes mêmes du sujet qui nous occupe en rendent compte: le statut du donneur; c'est dire que la loi n'envisage que le don. Deux conséquences en résultent immédiatement. La circulation à titre onéreux n'est pas autorisée, pas plus que la circulation à titre temporaire.

Tout d'abord, la loi prohibe la circulation à titre onéreux. À ce titre, les articles 16-5 et 16-6 du Code civil interdisent toute convention ayant pour effet de conférer une valeur patrimoniale à un élément du corps humain pour le premier, toute rémunération pour le prélèvement de ces mêmes éléments pour le second. Le Code de la santé publique fait écho à ce principe en indiquant qu' " [a]ucun paiement, quelle qu'en soit la forme, ne peut être alloué à celui qui se prête au prélèvement d'éléments de son corps ou à la collecte de ses produits ${ }^{15}$. C'est dire que l'altruisme est au cœur du statut du donneur, sa volonté est là encore paralysée par l'utilité sociale ; le législateur craint la dérive commerciale et le trafic de la matière humaine ${ }^{16}$. Et pour cause, une telle dérive commerciale, un tel trafic peuvent aboutir à des conditions de prélèvement susceptibles d'être contraires à la dignité humaine ${ }^{17}$. Par ailleurs, ils occasionneraient assurément une rupture dans l'égalité de traitement des receveurs: la vie pourrait devenir l'objet d'une marchandisation. Ceux qui disposeraient de moyens financiers importants pourraient plus facilement que les autres acquérir «le droit de continuer à vivre $»^{18}$. Enfin, une telle dérive inciterait les moins fortunés à faire commerce des éléments de leurs corps créant ainsi une véritable marchandisation du corps humain.

Ensuite, et bien que cela soit moins remarqué, le statut du donneur n'autorise que la circulation de la matière humaine à titre définitif. En effet, le livre II du Code de la santé publique est relatif au «don » et à l'utilisation des éléments et produits du corps humain et le premier texte de ce livre, l'article L. 1211-1, confirme cette lecture dans la mesure où il ne prévoit explicitement que la cession de matière humaine. Pourquoi le législateur n'a-t-il pas envisagé la mise à disposition temporaire? Faute d'intérêt? Il est permis d'en douter. Prenons un exemple: l'usufruit. Ce mécanisme est défini comme «le droit de jouir des choses dont un autre a la propriété, comme le propriétaire lui-même, mais à la charge d'en conserver la substance ${ }^{19}$. N'est-il pas légitime de penser que le «donneur» serait plus enclin à faire circuler un élément de son corps s'il avait une créance de restitution? Le receveur quant à lui n'en serait pas moins mal loti que dans l'hypothèse d'un don puisque l'usufruit ne cesse normalement qu'à la mort de l'usufruitier ${ }^{20}$. Mais au-delà de cette première remarque, la question se pose de savoir si le régime de l'usufruit est adapté aux buts que le législateur a assignés à la circulation de la matière humaine. Quid si le receveur altère la substance de cette matière? Il suffit d'imaginer un individu qui aurait bénéficié d'une greffe du poumon et qui se serait mis ou remis à fumer? Dans une telle hypothèse, le nu-propriétaire de l'organe devrait pouvoir agir en justice pour faire cesser l'atteinte à la substance. Dans cette perspective, il pourrait demander la restitution anticipée de la chose donnée en usufruit ${ }^{21}$. Une telle action

14. Art. L. 1211-1 du Code de la santé publique. On ajoutera que le texte évoque aussi les procédures judiciaires.

15. Art. L. 1211-4 du Code de la santé publique.

16. Voir A.-B. Caire, «Le corps gratuit...»; F. Granet-Lambrechts, «Les dons d'organes, de tissus, de cellules et de produits du corps humain: de la loi Caillavet aux lois de bioéthique», Revue de droit sanitaire et social, 1995, p. 1 sq.; R. Cabrillac, "Corps humain", in Dictionnaire des droits de l'homme, J. Andriantsimbazovina et al. (dir.), Paris, PUF, 2008: les «dérogations apportées au principe d'indisponibilité du corps humain sont enfermées dans des conditions strictes», dont fait partie «la gratuité, pour décourager l'apparition d'un marché des organes humains ».

17. L'impératif dépasse les frontières françaises. Voir notamment la Convention pour la protection des droits de l'homme et de la dignité de l'être humain à l'égard des applications de la biologie et de la médecine: Convention sur les droits de l'homme et la biomédecine, dite Convention d'Oviedo, signée le 4 avril 1997, ratifiée par la France le 13 décembre 2011 et opposable en droit interne depuis le $1^{\mathrm{er}}$ avril 2012.

18. J.-R. Binet, «La gratuité des éléments et produits du corps humain: entre esquive et faux semblant », in La gratuité, un concept aux frontières de l'économie et du droit, N. Martial-Braz, C. Zolynski (dir.), Paris, LGDJ, 2013, p. 263 sq., spéc. p. 270.

19. Art. 578 du Code civil.

20. L'article 617 du Code civil prévoit que l'usufruit prend fin, par principe, à la mort de l'usufruitier. Sur le caractère nécessairement temporaire de l'usufruit, voir F. Zenati-Castaing, T. Revet, Les biens, $3^{\mathrm{e}}$ éd., Paris, PUF, 2008, nº 301 : «Lorsqu'un bien est grevé d'un droit réel, la propriété s'en trouve affectée. [...] Le propriétaire ne peut opposer son pouvoir de manière absolue, puisqu'il doit tolérer l'existence d'un droit réel. Le caractère absolu de la propriété serait remis en cause si cette relativisation des prérogatives du propriétaire n'était pas temporaire. Dès lors que le titulaire d'un droit réel peut utiliser la chose et en tirer profit aussi durablement que le propriétaire, plus rien ne l'empêche de se dire lui-même propriétaire, encore que ses prérogatives soient différentes de celle d'un propriétaire. [...] La nature du régime actuel des biens commande donc que les droits réels soient temporaires». Voir aussi F. Danos, Propriété, possession et opposabilité, préface de L. Aynès, Paris, Economica, 20o7, $n^{\circ} 36$ : «La propriété ne peut s'accommoder de la coexistence d’un droit de jouissance perpétuel de tout ou partie des utilités de la chose qui priverait le propriétaire de sa vocation à jouir au moins potentiellement de l'intégralité des utilités de cette chose».

21. Art. 618 du Code civil: «L'usufruit peut aussi cesser par l'abus que l'usufruitier fait de sa jouissance, soit en commettant des dégradations sur le fonds, soit en le laissant dépérir faute d'entretien [...]. / Les juges peuvent, suivant la gravité des circonstances, ou prononcer l'extinction absolue 
en restitution est-elle compatible avec l'intérêt thérapeutique du receveur? Une réponse négative s'impose dans la mesure où elle est indissociable d'une atteinte à l'intégrité physique du receveur et susceptible de porter atteinte à sa vie... Ainsi, une opération faisant circuler de la matière humaine seulement à titre temporaire n'est pas compatible avec les objectifs fixés par la loi.

En droit positif, la conclusion apparaît avec évidence: l'individu est privé de toute initiative quant au choix du but et quant aux moyens relatifs à la circulation des éléments de son corps. Seul le don est envisageable, et encore seulement quand ce don répond aux objectifs fixés par la loi. Sans doute peut-on encore objecter à ce stade que le don est un contrat et que ce contrat repose essentiellement sur la volonté du donneur. Autrement dit, faute d'avoir le droit de cité quant à la destination des éléments du corps humain, la liberté et la propriété retrouveraient une place de choix à propos des modalités de l'opération juridique. Mais, là encore, il est difficile de souscrire à l'analyse au regard du droit positif.

\section{B. Les modalités des opérations autorisées au donneur}

L'absence de choix quant à la personne du receveur. Le donneur est-il libre de choisir le receveur? Une première lecture de la réglementation applicable au statut du donneur conduit à formuler une réponse négative. L'article L. 1211-5 du Code de la santé publique prévoit que le donneur ne peut connaître l'identité du receveur et réciproquement. En d'autres termes, le don est anonyme. Cet anonymat s'acclimate mal à la liberté contractuelle qui postule la liberté de choix du cocontractant. Pour tenter de contourner la difficulté, on pourrait avec certains auteurs considérer que l'acte juridique est conclu par le donneur, non pas avec le receveur, mais avec l'établissement hospitalier qui pratique le prélèvement ${ }^{22}$. Dans une telle perspective, le choix serait certes limité, mais il existerait. On serait bien en présence d'une liberté de principe confirmée par les restrictions motivées par l'intérêt général. Ces restrictions relatives au choix du cocontractant ne sont pas inconnues des juristes; on les retrouve dans de nombreuses opérations. On peut évoquer à cet égard la fiducie, contrat spécial qui réserve la qualité de fiduciaire à un établissement de crédit ou à un avocat ${ }^{23}$. On songe aussi aux opérations de banque qui ne peuvent être accomplies, à titre habituel, que par des établissements de crédit $^{24}$. Est-on dans une situation analogue en matière de don de matière humaine? On peut en douter dans la mesure où la loi n'envisage l'établissement de santé que comme un intermédiaire dans le cadre de la circulation d'un élément du corps humain. Il n'est pas le destinataire du don. Plus encore, l'opération n'est pas réglementée dans l'intérêt de l'établissement de santé, mais dans celui du receveur. En d'autres termes, il n'est pas possible d'exclure le receveur de l'opération. Est-ce à dire que la figure contractuelle est totalement exclue? Le droit des contrats connaît des mécanismes dans lesquels une personne accepte de contracter dans l'intérêt d'autrui. On pense bien sûr à la stipulation pour autrui ${ }^{25}$. Le donneur serait alors le stipulant et transmettrait la matière humaine à l'établissement de santé qui jouerait le rôle du promettant, à charge pour ce dernier de l'utiliser au profit du receveur qui serait le tiers bénéficiaire. On pourrait aussi y voir une espèce de fiducie, opération par laquelle le constituant - ici le donneur - transmet à un fiduciaire - l'établissement de santé - un des éléments de son corps à charge pour le fiduciaire de le gérer et de le rétrocéder à un tiers bénéficiaire, le receveur. Toutefois, toutes ces opérations nécessitent que le tiers bénéficiaire soit déterminé ou, à tout le moins, déterminable, ce qu'interdit le principe de l'anonymat. On le voit, l'anonymat paralyse bel et bien la liberté de choisir la personne au profit de laquelle une personne souhaite donner un élément de son corps.

Il ne reste alors qu'une manière pour tenter de déceler la liberté ou la propriété derrière le statut du donneur, mais non des moindres: le consentement, clé de voûte de tout acte juridique.

Le régime sui generis $d u$ consentement $d u$ donneur. Les actes juridiques se forment en principe sur le seul consentement libre et éclairé des parties. À la lecture des textes qui réglementent le statut du donneur, le consentement est au cœur de l'opération. Rien ne peut se faire sans le consentement du donneur. Peut-on y déceler l'expression de la liberté ou de la propriété en matière de don de matière humaine? On pourrait raisonnablement le soutenir. Toutefois, certains aspects de la réglementation applicable au

de l'usufruit, ou n'ordonner la rentrée du propriétaire dans la jouissance de l'objet qui en est grevé, que sous la charge de payer annuellement à l'usufruitier, ou à ses ayants cause, une somme déterminée, jusqu'à l'instant où l'usufruit aurait dû cesser ».

22. Voir G. Loiseau, «Le contrat de don d'éléments et produits du corps humain. Un autre regard sur les contrats réels», Recueil Dalloz, 2014, p. 2252 sq.

23. Art. 2015 du Code civil: «Seuls peuvent avoir la qualité de fiduciaires les établissements de crédit mentionnés au I de l'article L. 511-1 du code monétaire et financier, les institutions et services énumérés à l'article L. 518-1 du même code, les entreprises d'investissement mentionnées à l'article L. 531-4 du même code ainsi que les entreprises d'assurance régies par l'article L. 310-1 du code des assurances. / Les membres de la profession d'avocat peuvent également avoir la qualité de fiduciaire».

24. Art. L. 511-5 du Code monétaire et financier: «Il est interdit à toute personne autre qu'un établissement de crédit ou une société de financement d'effectuer des opérations de crédit à titre habituel. / Il est, en outre, interdit à toute personne autre qu'un établissement de crédit de recevoir à titre habituel des fonds remboursables du public ou de fournir des services bancaires de paiement».

25. Art. 1205 du Code civil: «On peut stipuler pour autrui. / L'un des contractants, le stipulant, peut faire promettre à l'autre, le promettant, d'accomplir une prestation au profit d'un tiers, le bénéficiaire. Ce dernier peut être une personne future mais doit être précisément désigné ou pouvoir être déterminé lors de l'exécution de la promesse». 
consentement du donneur suscitent des interrogations quant aux raisons pour lesquelles il occupe une place centrale en matière de don. Ces aspects concernent la faculté générale de révoquer son consentement d'une part et la présomption ponctuelle de consentement d'autre part.

Tout d'abord, le Code de la santé publique prévoit que le consentement du donneur est révocable à tout moment. En pratique, cela signifie que le consentement peut être retiré jusqu'au prélèvement de la matière humaine concernée. C'est dire que le concept d'offre ferme et définitive n'a pas cours en matière de don d'éléments du corps humain ${ }^{26}$; c'est dire aussi que l'exécution forcée ou la responsabilité contractuelle n'ont pas voix au chapitre. Autrement dit, le consentement tel qu'il est conçu en matière de don de matière humaine est largement dérogatoire à la manière dont on l'envisage lorsqu'il est question de faire circuler une chose. Certes, on peut être tenté de rapprocher le consentement en matière de don et en matière de donation. On sait en effet que le consentement du donateur est révocable à tout instant jusqu'à la remise de la chose ou l'acceptation de la donation devant notaire par le donataire. Toutefois, on explique cette révocabilité de la donation en droit des contrats par l'animus donandi, laquelle est indissociable de la personne du donataire ${ }^{27}$. C'est la raison pour laquelle l'erreur sur la personne ou sur les qualités de la personne est toujours une cause de nullité de la donation ${ }^{28}$. Or, s'agissant de don de matière humaine, le principe de l'anonymat exclut que l'auteur du don puisse connaître le receveur. La ratio legis de la révocabilité du consentement en matière de donation et en matière de don n'est donc pas la même. Et il est ainsi légitime de se demander si la révocabilité du prélèvement sans le consentement certain du donneur n'est pas davantage causée par le respect dû à son corps, l'inviolabilité de ce dernier, que par la liberté reconnue individuellement à chacun de nouer une relation contractuelle.

Ensuite, le consentement du donneur est dans certaines circonstances présumé. Il en va ainsi en ce qui concerne le prélèvement d'organes sur une personne décédée; cette présomption ne tombe en principe que si l'individu a indiqué sa volonté de ne pas subir de prélèvement suite à sa mort. Mais le législateur, sentant sans doute le risque de dérive lié à une telle présomption, s'est empressé de la nuancer en indiquant que le médecin doit, si le de cujus n'est pas inscrit sur la liste, questionner ses proches pour entériner la présomption ${ }^{29}$.
Que penser de ces mécanismes au regard de la liberté individuelle de faire circuler les éléments de son corps? La liberté contractuelle correspond à la liberté de s'obliger et cette liberté est prise à défaut par les mécanismes encadrant le consentement en matière de don; révocable, le consentement n'oblige pas, c'est la tradition qui, en vertu de la loi et pour des raisons qui nous semblent différentes de celles qui animent son traitement juridique en matière de donation, rend le don irrévocable. Ce sentiment est renforcé par la présomption de consentement. Présumé, ce n'est pas le consentement qui oblige; là encore, c'est la loi qui oblige.

Ainsi, si la loi présente le consentement comme révocable, si la loi présente le consentement comme pouvant être présumé, ce n'est pas en raison de la liberté individuelle, mais une fois de plus en raison de l'utilité publique: dans le premier cas, c'est la dignité de la personne qui est en cause, l'inviolabilité du corps humain. Dans le second cas, c'est l'intérêt thérapeutique qui est en cause: il faut augmenter autant que faire se peut le nombre de greffons disponibles.

Bien que le Code de la santé publique organise la circulation des éléments du corps humain, que cette circulation est indissociable de la reconnaissance, dans notre système juridique, du concept de propriété des éléments du corps humain, de liberté contractuelle à leur égard, la tentative d'application de ces concepts échoue en droit positif. La réalité du paradigme est différente: là où on enseigne habituellement que les choses circulent librement sous réserve de ne pas contrevenir à l'intérêt général, les éléments du corps humain circulent en raison de l'intérêt général sous réserve de ne pas contrevenir à d'autres impératifs d'ordre général. Le schéma établi ne laisse à la liberté et à la propriété qu'une place résiduelle, celle d'un instrument de correction permettant à l'individu d'interdire plus que de choisir.

\section{La mise à l'épreuve du paradigme reposant sur l'intérêt général}

En droit positif, la circulation des éléments du corps n'est pas causée par la propriété ou la liberté, elle est causée par l'intérêt général. La réglementation du statut du donneur tente de réaliser un compromis entre dignité humaine et intérêt scientifique ou thérapeutique. Mais le résultat est loin d'être satisfaisant (A). Un tel constat autorise à s'interroger sur le point de savoir s'il est pertinent de

26. Art. 1114 du Code civil: «L'offre, faite à personne déterminée ou indéterminée, comprend les éléments essentiels du contrat envisagé et exprime la volonté de son auteur d'être lié en cas d'acceptation. À défaut, il y a seulement invitation à entrer en négociation".

27. Voir J. B. de Saint-Affrique, in Jurisclasseur Code civil, fascicule unique, art. 901, $\$ 21$.

28. Voir Cass., $1^{\text {re }}$ civ., 13 avril 1964 , Bulletin civil $I, \mathrm{n}^{\circ} 185$.

29. Art. L. 1232-1 du Code de la santé publique: «Le prélèvement d'organes sur une personne dont la mort a été dûment constatée ne peut être effectué qu'à des fins thérapeutiques ou scientifiques. / Le médecin informe les proches du défunt, préalablement au prélèvement envisagé, de sa nature et de sa finalité, conformément aux bonnes pratiques arrêtées par le ministre chargé de la santé sur proposition de l'Agence de la biomédecine [voir aussi, sur l'atteinte portée aux droits des proches parents lorsque le médecin ne les consulte pas: Cour EDH, 24 juin 2014, Petrova c. Lettonie, $\mathrm{n}^{\circ}$ 4605/05; J.-P. Marguénaud, "Menaces sur la règle du consentement présumé au prélèvement d'organes post mortem», Revue trimestrielle de droit civil, 2014, p. 840]./ Ce prélèvement peut être pratiqué sur une personne majeure dès lors qu'elle n'a pas fait connaître, de son vivant, son refus d'un tel prélèvement, principalement par l'inscription sur un registre national automatisé prévu à cet effet. Ce refus est révocable à tout moment. / L'Agence de la biomédecine est avisée, préalablement à sa réalisation, de tout prélèvement à fins thérapeutiques ou à fins scientifiques ». 
laisser la liberté et la propriété n'intervenir que de manière accidentelle en la matière (B).

\section{A. Un compromis hasardeux}

L'intérêt général. L'intégralité des questions relatives au statut du donneur est commandée par l'intérêt général et plus exactement par deux intérêts collectifs inconciliables. Le législateur s'est à cet égard enfermé dans un système contradictoire. En proclamant l'inviolabilité du corps humain, le respect qui lui est dû, l'impossibilité d'en faire l'objet d'un droit patrimonial, il a érigé en principe général la protection de la personne humaine dans sa dignité contre toutes formes de trafic et de pratiques odieuses. Ce principe annihile la liberté de la personne quant à la circulation des éléments de son corps. Mais, dans le même temps, le législateur a poursuivi un autre objectif: parce que la circulation des éléments du corps humain a des bénéfices considérables quant à la préservation de la vie de la personne humaine dont la primauté doit être assurée, il a, de manière dérogatoire, organisé sa circulation. Autrement dit, lorsque la circulation est nécessaire à des fins scientifiques ou thérapeutiques, elle est autorisée et réglementée par le législateur sous réserve de ne pas porter une atteinte trop importante au respect dû au corps humain. Telles sont les données de l'équation en droit positif qui éclairent tout le régime du don de matière humaine. Ces deux impératifs, ces deux intérêts collectifs sont la matrice de toutes les règles relatives au statut du donneur. On peut, pour l'illustrer, prendre deux exemples emblématiques de ces règles: l'anonymat et la gratuité du don.

L'anonymat. Le don d'organes et de tissus est un geste altruiste et fondé sur la solidarité humaine. Pour garantir ce principe, le législateur a considéré que le don devait être anonyme. Parce que le législateur entend protéger la personne dans sa dignité, assurer le respect dû au corps humain, le don est anonyme ${ }^{30}$. En interposant un organisme hospitalier qui fait écran entre le donneur et le receveur, il garantit autant que faire se peut que l'individu ne sera pas tenté d'organiser une marchandisation des éléments de son corps. Il garantit aussi que les proches d'un receveur ne pourront pas exercer de pression sur le consentement du donneur. Toutefois, lorsque l'intérêt thérapeutique le nécessite, l'anonymat est remis en cause. Tel est le cas en matière de don d'organe par un donneur vivant. Dans la mesure où le don d'organe est, pour un individu vivant, une opération à risque, la loi est venue restreindre le nombre de personnes pouvant effectivement se positionner comme donneur d'organe. L'idée est la suivante: une personne ne peut donner de son vivant que si le don est causé. En d'autres termes, c'est l'intérêt thérapeutique du donneur qui commande que l'on vérifie la raison pour laquelle il donne et qui fonde la levée de l'anonymat. Ainsi, l'article L. 1231-1 du Code de la santé publique prévoit que peut seul être donneur, dans l'intérêt thérapeutique du receveur, un membre de sa famille ou une personne ayant avec lui un lien affectif étroit et stable depuis au moins deux ans. La rédaction du texte appelle deux remarques.

Initialement, le don d'organe par une personne vivante n'était possible que dans le cercle familial. Ce n'est qu'en 2011 que le législateur a ouvert cette faculté aux personnes présentant un lien affectif étroit et stable avec le receveur. Faut-il y voir une victoire de la liberté individuelle, celle de choisir son cocontractant? Certainement non. La lecture des travaux préparatoires est sur ce point univoque. C'est en raison de la pénurie d'organe que la loi a élargi le cercle des donneurs potentiels ${ }^{31}$. En d'autres termes, la matrice de l'évolution de la règle posée à l'article L. 1231-1 du Code de la santé publique n'est pas la liberté de l'individu, mais bien l'intérêt thérapeutique du receveur.

L'évolution du texte montre aussi que l'arbitrage que doit réaliser la loi ne met pas toujours en cause d'une part la dignité de la personne humaine et d'autre part l'intérêt thérapeutique ou scientifique. L'article L. 1231-1 du Code de la santé publique montre que, parfois, le compromis opéré par la loi met en scène l'intérêt thérapeutique du donneur d'un côté et celui du receveur de l'autre côté.

La gratuité. Là encore, la gratuité s'impose pour éviter toute marchandisation du corps humain. C'est donc la dignité et le respect du corps humain qui en sont le fondement. Mais l'intérêt thérapeutique conduit à inciter de manière de plus en plus pressante les personnes à réaliser des dons. Dès lors, de manière directe ou indirecte, la gratuité est remise en cause pour augmenter le nombre de dons. Tel est le cas par exemple en matière d'ovocytes. En principe, une femme ne peut avoir recours au prélèvement et à la conservation de ses gamètes dans son propre intérêt que pour des raisons médicales. Le nombre de gamètes données n'étant pas assez important, le législateur a pris une résolution qui n'est pas réellement compatible avec la gratuité. Il a ouvert aux femmes qui n'ont pas encore procréé le droit de donner leurs ovocytes et, pour les y inciter, il a prévu qu'une partie des ovocytes prélevés dans une telle hypothèse peut être conservée à des fins autologues ${ }^{32}$. En d'autres termes, la

30. Art. L. 1211-5 du Code de la santé publique: «Le donneur ne peut connaître l'identité du receveur, ni le receveur celle du donneur. Aucune information permettant d'identifier à la fois celui qui a fait don d'un élément ou d'un produit de son corps et celui qui l'a reçu ne peut être divulguée. / Il ne peut être dérogé à ce principe d'anonymat qu'en cas de nécessité thérapeutique».

31. Voir J. Penneau, "Corps humain - Bioéthique», Répertoire de droit civil, 2012 (actualisation avril 2017), \$169 et 170.

32. Art. L. 1244-2 du Code de la santé publique: «Le donneur doit avoir procréé. Le consentement des donneurs et, s'ils font partie d'un couple, celui de l'autre membre du couple sont recueillis par écrit et peuvent être révoqués à tout moment jusqu'à l'utilisation des gamètes. / Il en est de même du consentement des deux membres du couple receveur./Lorsqu'il est majeur, le donneur peut ne pas avoir procréé. Il se voit alors proposer le recueil et la conservation d'une partie de ses gamètes ou de ses tissus germinaux en vue d'une éventuelle réalisation ultérieure, à son bénéfice, d'une assistance médicale à la procréation, dans les conditions prévues au titre IV du livre Ir de la deuxième partie. Ce recueil et cette conservation sont subordonnés au consentement du donneur». 
donneuse bénéficie d'une contrepartie directe et immédiate qu'elle n'aurait pas pu obtenir autrement, c'est-à-dire sans avoir procédé au don ${ }^{33}$.

L'atteinte portée au principe de gratuité du don est parfois plus discrète; elle n'apparaît qu'indirectement, mais cela ne signifie pas qu'elle n'existe pas. On en prendra deux exemples qui là encore sont motivés par l'intérêt thérapeutique.

Tout d'abord, le législateur a autorisé la pratique des dons croisés, ce qui prend quelques peu à défaut l'altruisme du don ${ }^{34}$. Dans une telle hypothèse, une personne accepte de donner son organe à une autre qu'elle connaît. Toutefois, le donneur et le receveur ne sont pas compatibles, mais le donneur est compatible avec un autre receveur. Ce second receveur a lui-même un donneur avec lequel il n'est pas compatible, mais qui est compatible avec le premier receveur. Ainsi, le premier donneur donne au second receveur et le second donneur donne au premier receveur. Dans un tel montage, le donneur n'est plus totalement désintéressé : il donne à une personne pour obtenir d'une autre personne qu'elle donne également au receveur initial avec lequel il n'est pas compatible.

Ensuite, comme cela a été évoqué, le législateur a élargi le cercle des donneurs vivants en matière de don d'organe ${ }^{35}$. Dorénavant, toute personne qui présente un lien affectif étroit et stable avec le receveur depuis au moins deux ans peut être donneur. Comment ne pas voir le risque de dérive lié à cet élargissement? Comment ne pas imaginer que les receveurs ayant quelques moyens financiers puissent, aujourd'hui, trouver un arrangement avec une personne compatible et créer de manière artificielle le lien affectif durable et stable pour satisfaire à l'exigence pour le moins souple posée par le législateur ${ }^{36}$ ?

Ces différents exemples montrent que la marchandisation des éléments du corps humain dans la relation donneurreceveur pénètre de plus en plus dans le donné, elle devient un fait qu'il est difficile d'occulter d'autant plus que le droit en est devenu, au nom de l'intérêt thérapeutique, une cause non négligeable. Un tel constat n'invite-t-il pas à revisiter la place accordée à la liberté individuelle et à la propriété en matière de don de matière humaine? Encore faut-il, pour l'envisager, sortir ces prérogatives du carcan dans lequel elles ont été enfermées, celui d'un simple instrument de correction.

\section{B. La liberté et la propriété: des instruments de correction aujourd'hui, des matrices du statut du donneur demain?}

En droit positif, les prérogatives individuelles de la personne sur les éléments de son corps se résument à un choix: je donne ou je ne donne pas. La liberté individuelle et la propriété, présentées comme des principes cardinaux de notre droit, ne peuvent pas s'épanouir sur la base d'un choix binaire. Une chose est de dire que l'on ne peut contraindre une personne à donner, une autre de reconnaître qu'elle est libre de choisir ce qu'elle veut faire des éléments de son corps. Dès lors, si l'on veut faire de la liberté individuelle et de la propriété des matrices d'une nouvelle réglementation du statut du donneur, il ne suffit pas de les prendre en considération à côté de la dignité humaine et de l'intérêt thérapeutique et scientifique. Il faut rétablir la hiérarchie voulue par les auteurs de la DDHC et du Code civil. L'individu doit être replacé au cœur du système. Pour cela, il est nécessaire de faire table rase de la réglementation existante et de la réécrire en proclamant la propriété des éléments du corps humain comme étant le principe et l'intérêt général quel qu'il soit comme le fondement des restrictions ponctuelles à la circulation des éléments du corps. Plusieurs arguments plaident en faveur d'une telle reconstruction.

Tout d'abord, la crainte de la marchandisation des éléments du corps humain qui paralyse la liberté individuelle et la propriété au nom de la dignité humaine a déjà pénétré la pratique. Le sang a un prix même si celui qui le produit ne peut en tirer profit. La loi réglemente les échanges entre les établissements de santé et fixe par arrêté la valeur patrimoniale du sang ${ }^{37}$. Elle organise aussi l'import et l'export des organes ${ }^{38}$. Et on a vu que, même dans la relation donneur-receveur, l'intérêt thérapeutique conduit aujourd'hui à créer les conditions d'une patrimonialisation ostensible ou occulte des éléments du corps humain ${ }^{39}$. N'est-il pas temps de mettre un terme à l'hypocrisie du système et de réglementer la circulation des éléments du corps humain autrement qu'en opposant systématiquement le respect dû au corps humain et l'intérêt thérapeutique? La liberté individuelle et la propriété ne sont-elles finalement pas une alternative à un système qui repose sur la confrontation de deux fondements et dont les règles qui en sont issues ne sont finalement totalement satisfaisantes ni à l'égard du premier, ni à l'égard du second?

33. Voir J.-R. Binet, «La gratuité des éléments et produits du corps humain...», spéc. p. 271.

34. Art. L. 1231-1, al. 3 du Code de la santé publique: «En cas d'incompatibilité entre la personne ayant exprimé l'intention de don et la personne dans l'intérêt de laquelle le prélèvement peut être opéré en vertu des premier ou deuxième alinéas, rendant impossible la greffe, le donneur et le receveur potentiels peuvent se voir proposer le recours à un don croisé d'organes. Celui-ci consiste pour le receveur potentiel à bénéficier du don d'une autre personne ayant exprimé l'intention de don et également placée dans une situation d'incompatibilité à l'égard de la personne dans l'intérêt de laquelle le prélèvement peut être opéré en vertu des premier ou deuxième alinéas, tandis que cette dernière bénéficie du don du premier donneur. En cas de mise en œuvre d'un don croisé, les actes de prélèvement et de greffe sont engagés de façon simultanée respectivement sur les deux donneurs et sur les deux receveurs. L'anonymat entre donneur et receveur est respecté ».

35. Voir supra.

36. Voir J.-R. Binet, «La gratuité des éléments et produits du corps humain...», spéc. p. 270.

37. Arrêté du 9 mars 2010 relatif au tarif de cession des produits sanguins labiles.

38. Voir notamment les articles L. 1235-1 et R. 1221-68 du Code de la santé publique.

39. Voir supra. 
Ensuite, et dans la veine du premier argument, la liberté individuelle et la propriété sont des concepts éprouvés; à leur égard, la technique juridique existe et peut parfaitement s'adapter à la particularité des choses que sont les éléments du corps humain. Il suffit de constater que la propriété et le contrat sont déjà grandement sollicités à propos des cheveux, des ongles ou des dents que le législateur a laissés en marge de la réglementation du statut du donneur ${ }^{40}$.

Enfin, le législateur est conscient que le statut du donneur est une matière en constante évolution. Il a ainsi prévu que les lois sur la bioéthique devraient être rediscutées tous les sept ans ${ }^{41}$. Cela tient me semble-t-il à trois facteurs. Le premier concerne les avancées scientifiques qui permettent de développer de nouvelles techniques thérapeutiques. Le deuxième est le contrôle de l'efficience des dispositifs mis en place dans l'intérêt thérapeutique de l'individu. Le dernier est sans doute celui qui nous intéresse le plus en ce qu'il pourrait permettre de remettre la réglementation du statut du donneur à plat: l'appréciation des valeurs sociales change, les mœurs évoluent et il n'est pas impossible que l'axiome qu'est l'inviolabilité du corps humain cède face à la liberté de la personne sur les éléments de son corps en particulier et sur son corps en général.

Peut-on aller plus loin? Selon nous, la réponse est non. Il appartient aux juristes de dire ce qu'est la liberté, ce qu'est la propriété; il appartient au juriste de montrer les dysfonctionnements et les contradictions qui affectent le statut du donneur; il appartient au juriste de dire si, techniquement, il est possible d'organiser la circulation des éléments du corps humain sur le fondement de la liberté individuelle et de la propriété. Mais il n'appartient pas au juriste de décider si le statut du donneur doit avoir pour axiomes la liberté individuelle et la propriété à la place de l'inviolabilité du corps humain et de l'intérêt thérapeutique; cette décision est, par essence, politique.

40. Art. R. 1211-49 du Code de la santé publique.

41. Art. 47 de la loi n $2011-814$ du 7 juillet 2011: «I. La présente loi fait l'objet d'un nouvel examen d'ensemble par le Parlement dans un délai maximal de sept ans après son entrée en vigueur./ II. Elle fait en outre l'objet, dans un délai de six ans, d'une évaluation de son application par l'Office parlementaire d'évaluation des choix scientifiques et technologiques». 